\title{
Topology and Energetics of Metal-Encapsulating Si Fullerene-Like Cage Clusters
}

\author{
Takehide Miyazaki $^{a}$, Hidefumi Hiura ${ }^{b}$ and Toshihiko Kanayama ${ }^{a}$ \\ ${ }^{a}$ Advanced Semiconductor Research Center, National Institute of Advanced Industrial Science and \\ Technology, AIST Tsukuba Central 4, \\ 1-1-1 Higashi, Tsukuba 305-8562, Japan \\ ${ }^{b}$ Fundamental Research Laboratories, NEC Corporation, \\ 34 Miyukigaoka, Tsukuba 305-8501, Japan
}

(October 29, 2018)

\begin{abstract}
On the basis of a topological discussion as well as an ab initio calculation, we show that it is possible to construct a fullerene-like Si cage by doping of a transition metal atom. The cage is a simple 3-polytope which maximizes the number of its inner diagonals close to the metal atom. Our topological argument also reveals how closely the structure of the fullerene-like Si cages studied is related to that of fullerenes themselves.
\end{abstract}

PACS numbers: 31.10.+z, 36.40.-c, 36.40.Qv, 61.48.+c

Typeset using REVTEX 
Synthesis of fullerene-cage-like clusters composed of elements other than carbon (C) is a subject of great interest [1]. Especially, it is intriguing to study whether silicon (Si) analogues of the fullerenes can exist in energetically favorable forms. Production of Si cage clusters is also important from a technological point of view. They may be used as building blocks for fabrication of various structures in electronic devices in ten nanometer scales.

A main question which we would like to answer in this study is how one can obtain a smooth fullerene-like cage composed of Si atoms. Due to their $s p^{3}$ nature, the Si atoms tend to bind themselves against generation of fullerene cages. As a matter of fact, $\mathrm{Si}_{n}$ clusters $(n$ up to $\sim 50$ ) usually favor compact forms which are completely different from fullerene cages [2].3]. The structure of a $\mathrm{Si}_{60}$ cluster in a fullerene cage is highly distorted [4].

As is well known, the structure of a typical fullerene cage $\left(\mathrm{C}_{n}\right)$ is composed of only 5and 6-membered rings. The number of the 5-membered rings is twelve irrespective of $n$. Each atom is connected with three neighbors. Stability of fullerenes may be attributed to two factors: $\sigma$ bonding among $s p_{\|}^{2}$ hybrids, where $p_{\|}$stands for the intra-cage component of the $\mathrm{C} p$ orbital, and $\pi$ conjugation among $p$-orbital components normal to the cage surface $\left(p_{\perp}\right)$.

A fundamental difficulty to maintain a smooth Si fullerene cage stems from the fact that the $\pi$ conjugation among the $p_{\perp}$ orbitals does not occur. Thus, the caging mechanism is only the $\sigma$ bonding of the $s p_{\|}^{2}$ hybrids. The distortion of the fullerene cage of $\mathrm{Si}_{60}$ is due to admixture of substantial $p_{\perp}$ components with $s p_{\|}^{2}$.

A promising solution to the augmentation of Si cage structures is to put one or more additional atoms inside of the cages. If electron orbitals of these "doped" atoms have a substantial overlap with the $p_{\perp}$ orbitals, then the additional cohesion forces would be supplied to the cage. This idea has originally sprouted from the construction of $\mathrm{Si}_{n}$ clusters with $n$ larger than $\sim 20$ [5] to account for the exceptional chemical inertness of $\mathrm{Si}_{39}$ and $\mathrm{Si}_{45}$ [6] and also for "prolate-oblate" structural change in $\mathrm{Si}_{n}$ around $n \sim 27$ [7]. A common aspect of the $\mathrm{Si}_{n}$ configurations studied in Refs. [5] is that they are configured to mimic as much of the bulk Si structure as possible, while the outermost cages yet resemble the counterparts of fullerenes.

As explained above, there are at least two mechanisms to stabilize a doped Si fullerenecage cluster: (a) $\sigma$ bonding within the $s p_{\|}^{2}$ hybrid network of the cage and (b) bonding among the $p_{\perp}$ orbitals of the cage and the orbitals of doped atoms. A key issue to be solved for production of stable fullerene-like Si cage clusters is how these two factors should be tuned in order to maximize the total binding among the constituent atoms in the clusters.

The goal of this study is to give an explicit answer to this question from a theoretical point of view. For this purpose, we target metal-doped Si cage clusters, $M @ \mathrm{Si}_{n}$, because we have recently experimentally suggested that stable metal-encapsulating Si cage clusters $\left(M @ \mathrm{Si}_{n}, M=\mathrm{Hf}, \mathrm{Ta}, \mathrm{W}, \mathrm{Re}, \mathrm{Ir}\right.$, etc. and $\left.n \leq \sim 14\right)$ might be grown 8]. We also note the outstanding property of $M @ \mathrm{Si}_{n}$. When doped with $\mathrm{Na}$ and $\mathrm{Ba}$, the $M @ \mathrm{Si}_{n}$ clusters in certain phases of the solid state exhibit superconductivity [9]. Although some theoretical [10] as well as experimental [11] studies have been performed for metal-doped Si clusters, roles of the cage topologies played in stabilization of fullerene-like Si cages have been little argued.

First, we discuss what topology should be suitable for a cage of $M @ \mathrm{Si}_{n}(n \leq 20)$. A very important clue to the solution has been recently given by mathematicians, Bremner 
and Klee [12]. They study inner diagonals (see below or Ref. [12] for definition of an inner diagonal) of convex polytopes. The quantity of our own interest here is the number of inner diagonals denoted by $\delta_{3}$. We will propose that a simple 3-polytope [13], in which the number of inner diagonals close to the dopant $\left(\delta_{3}^{\text {eff }}\right)$ is maximized, should be a candidate of a stable fullerene-like Si cage of $M @ \mathrm{Si}_{n}$.

Next, we perform a first-principles energetics of the clusters in the case of $M=\mathrm{W}$ (W@Si ${ }_{n}$, $8 \leq n \leq 16)$. The clusters in fullerene-like cages predicted by the above topological picture are indeed obtained for $n=12$ [Fig.1(d)] and $n=14$ [Fig.1(e)]. For $n=8$ and 10, however, the caging with corresponding simple 3-polytopes [Fig.1(a) and Fig.1(b)] may not occur, because their total energies are significantly higher than those of the respective isolated atoms. For $n=16$, a single cage is unstable (Fig.2). The cage is relaxed into a skeleton similar to either of $n=12$ [Fig.1(d)] or $n=14$ [Fig.1(e)]. Thus we conclude that it is possible to construct energetically favorable fullerene-like Si cage clusters doped with a transition metal atom, whose cage sizes depend on the dopant.

Now we describe our results. First a topological discussion of Si cages is given. We denote the number of $p$-membered rings of a $\mathrm{Si}$ cage as $N_{p}$. Throughout this study, we limit the range of $p$ to $4 \leq p \leq 6$. We also use a vector notation $\vec{N}=\left(N_{4}, N_{5}, N_{6}\right)$ for brevity. For example, simple 3 -polytopes with $n=8$ and 10 vertices are a cube $[\vec{N}=(6,0,0)$, Fig.1(a) [14]] and a pentagonal prism $[\vec{N}=(5,2,0)$, Fig.1(b)], respectively. For each $n \geq 12$, simple 3 -polytopes with different $\vec{N}$ 's exist as shown in Fig.1 and Table I [15].

An inner diagonal (or a 3-diagonal) of a convex 3-polytope $P$ is defined as a segment that joins two vertices of $P$ and that lies, except for its ends, in $P$ 's relative interior [12]. There are two other kinds of diagonals of $P, 1$ - and 2-diagonals. The former are the edges. As for the latter, there are two, five and nine 2-diagonals in a 4-, 5- and 6-membered ring, respectively. If we denote the number of $i$-diagonals by $\delta_{i}$, then $\delta_{1}+\delta_{2}+\delta_{3}=\frac{n(n-1)}{2}$, where $n$ is the number of vertices. For example, $\delta_{3}=4$ for $\vec{N}=(6,0,0)$ and $\delta_{3}=10$ for $\vec{N}=(5,2,0)$. In this study, we further define $\delta_{3}^{e f f}$ as $\delta_{3}^{e f f}=\sum_{i>j} \theta\left(d_{\text {cut }}-d_{i j}\right)$, where $i$ and $j$ are indices of Si atoms, $d_{\text {cut }}$ is the cut-off radius and $d_{i j}$ is the distance between a metal atom and a diagonal joining the $i$-th and $j$-th Si atoms. $\theta(x)$ is a step function; $\theta(x)=1$ for $x \geq 0$ and $\theta(x)=0$ otherwise. Obviously, $\delta_{3}^{\text {eff }}$ approaches $\frac{n(n-1)}{2}$ for $d_{\text {cut }} \rightarrow \infty$. In order to count the number of only inner diagonals, $d_{\text {cut }}$ must be small. In practice, we vary $d_{\text {cut }}$ from $1.0 \AA$ down to $0.25 \AA$ to check the $d_{\text {cut }}$-dependence on $\delta_{3}^{e f f}$. The overlap between the $p_{\perp}$ orbitals of a $\mathrm{Si}$ cage and the counterparts of the dopant metal atom may be roughly proportional to the number of the $p_{\perp}$ orbitals pointing toward the metal atom, which should also be approximately proportional to $\delta_{3}^{\text {eff }}$ at a small $d_{\text {cut }}$ by its definition. Thus analyzing $\delta_{3}^{\text {eff }}$ may measure the strength of the bonding between the doped atom and the Si cage. Our first-principles calculation shows that $\delta_{3}^{e f f}$ at $\delta_{\text {cut }}=0.25 \AA$ is maximized for the lowest-energy $\mathrm{W} @ \mathrm{Si}_{n}$ cluster for each of $n=12$ and 14.

Bremner and Klee [12] show that, for a given $n, P$ with a maximum $\delta_{3}$ is simplicial [13]. A simplicial 3-polytope seems the best for a cage of $M @ \mathrm{Si}_{n}$ for each $n$, because the energy gain due to the factor (b) should be fully enjoyed. This is not true, however, since the mechanism (a) is instead sacrificed so that a total energy gain is not maximized. Our first-principles calculation shows that a $\mathrm{W}_{0} \mathrm{Si}_{12}$ cluster with an icosahedron cage (i.e. a simplicial 3-polytope with 12 vertices and $\delta_{3}=36$ ) is energetically very unfavorable [5.76 $\mathrm{eV}$ higher in energy than the (6,0,2)-cage cluster shown in FIg.1(d)], despite that all $p_{\perp}$ 
orbitals of the cage point toward the central W atom. The averaged Si-Si distance $\left(d_{2}\right)$ of the icosahedron cage $(2.72 \AA)$ is much longer than those of both $(4,4,0)-\left(d_{2}=2.42 \AA\right)$ and $(6,0,2)$-cages $\left(d_{2}=2.39 \AA\right)$. In contrast, the averaged Si-W distances $\left(d_{1}\right)$ are similar, $\sim 2.6 \AA$. This evidences that too much preference of the factor (b) is taken over (a) in the icosahedron cage. Therefore a simple 3-polytope is a good candidate for a $\mathrm{Si}_{n}(n \leq 20)$ cage, in which both (a) and (b) factors may work equally well to enhance the stability of the $M @ \mathrm{Si}_{n}$ cluster as a whole.

Turning to the topology of a carbon fullerene cage, its peculiarity can be characterized in light of $\delta_{3}$. Let $Q$ be a simple 3-polytope with $n$ vertices. For each of $n \geq 24, Q$ with $F$ $(=n / 2+2)$ facets composed of only twelve pentagons and $F-12$ hexagons maximizes $\delta_{3}$ which is $n(n-13) / 2+30$ [16]. For $n=20, Q$ with the largest $\delta_{3}(=100)$ is a dodecahedron which has only twelve pentagons. In the case of $n=22$, however, $Q$ with $N_{5}=12$ cannot be realized [12. The maximum possible value of $\delta_{3}$ is 128 when $\vec{N}=(1,10,2)$. Thus a typical fullerene $\mathrm{C}_{n}$ cage $(n \geq 20)$ can be identified to be a simple 3 -polytope with a maximum value of $\delta_{3}$ except $n=22$. The $\mathrm{Si}_{n}$ cages we consider for $8 \leq n \leq 16$ include $Q$ 's with maximum $\delta_{3}$ 's. Although $\delta_{3}(=18)$ of the $(6,0,2)$-cage [Fig.1(d)] is not a maximum, it is the second largest value. This is why we can say that our Si cages are fullerene-like.

In order to substantiate the above topological argument, we perform a first-principles energetics of the $\mathrm{W} @ \mathrm{Si}_{n}$ clusters. Here we adopt a recently proposed single-parent evolution algorithm (SPEA) [17] to update the atomic coordinates in an unbiased way. For SPEA simulation, we calculate the total energies of clusters with a linear combination of atomic orbitals (LCAO) using the GAUSSIAN98 package 18. Then we use the calculated coordinates of the clusters as the inputs of plane-wave (PW [19]) total energy calculations [20] where quenched molecular dynamics is performed for final convergence. Electronic structures of clusters are calculated with density-functional theory 21] within generalized gradient approximation to the exchange-correlation energy functionals (Becke'88 [22] and Perdew-Wang'91 [23] for LCAO and Perdew-Wang-Ernzerhof'96 [24] for PW) [25].

With the aid of SPEA, we indeed find that the cages of energetically favorable W@ $\mathrm{Si}_{12}$ and W@Si ${ }_{14}$ clusters are simple 3-polytopes with $\vec{N}=(6,0,2)$ [Fig.1(d)] and (3,6,0) [Fig.1(e)], respectively. Varying $d_{\text {cut }}$ from $1.0 \AA$ down to $0.25 \AA$, we find that $\delta_{3}^{\text {eff }}$ 's of both are constants ( 6 and 4; see Table I for the summary of the result). On the other hand, $\delta_{3}^{e f f}$ 's of the other $\mathrm{W} @ \mathrm{Si}_{12}$ and $\mathrm{W} @ \mathrm{Si}_{14}$ clusters decrease to $0-2$ as $d_{\text {cut }}$ becomes smaller, whose total energies are all higher than those of the $(6,0,2)$ and $(3,6,0)$ cages, respectively. These results support the topological argument made above.

It should be noted that the $\mathrm{W} @ \mathrm{Si}_{n}(n \sim 10)$ clusters may be viewed as metastable phases of Si-rich tungsten silicides, which do not appear in equilibrium W-Si phase diagram [26]. It is suggested in the previous experiment [8] that the clusters are obtained via a sequential growth where a $\mathrm{W} @ \mathrm{Si}_{n}$ cluster is obtained by attaching additional $m$ Si atoms to a smaller $\mathrm{W} @ \mathrm{Si}_{n-m}$ cluster. In order to understand the essence of the growth process on the basis of our energetics, we calculate differential binding energy, defined as $\Delta E(n)=E_{\mathrm{bind}}(n+$ $2)-2 E_{\text {bind }}(n)+E_{\text {bind }}(n-2)$, where $E_{\text {bind }}(n)=E_{\text {total }}^{\text {atom }}(\mathrm{W})+n E_{\text {total }}^{\text {atom }}(\mathrm{Si})-E_{\text {total }}^{\text {cluster }}\left(\mathrm{WSi}_{n}\right) . \mathrm{A}$ negative value of $\Delta E(n)$ means that generation of a W@Si ${ }_{n}$ cluster is favorable. We find that $\Delta E(10)=1.6 \mathrm{eV}, \Delta E(12)=-2.8 \mathrm{eV}$ and $\Delta E(14)=-0.3 \mathrm{eV}$. For $n=10$, stable caging of a $\mathrm{W}$ atom in simple 3-polytopes [Fig.1(b)] is unlikely to occur. The cluster should be chemically very reactive, because there are too few $\mathrm{Si}$ atoms around $\mathrm{W}$ to fulfil its reaction 
points. If additional $\mathrm{Si}$ atoms can arrive at the cluster prior to its collapse, then subsequent growths to $\mathrm{W} @ \mathrm{Si}_{12}$ and possibly to $\mathrm{W} @ \mathrm{Si}_{14}$ should occur with high probabilities.

We also find that there exists a threshold cage size for $\mathrm{W} @ \mathrm{Si}_{n}$ beyond which the cages become unstable. In Fig.2, some of calculated structures of $\mathrm{W} @ \mathrm{Si}_{16}$ clusters are shown. The initial configurations of the cages are taken like simple 3-polytopes with 16 vertices. Upon relaxation, all cages we considered are completely distorted and become similar to either the $(6,0,2)$ cage plus $2 \mathrm{Si}$ atoms or the $(3,6,0)$ cage plus $4 \mathrm{Si}$ atoms. It appears that $n=14$ is the threshold size in the case of $\mathrm{W}$ doping. For a cage beyond a critical size, there are too many $\mathrm{Si}$ atoms around $\mathrm{W}$ to cover its reaction points.

In conclusion, we show that it is possible to construct $\mathrm{Si}$ clusters in fullerene-like cages with transition-metal atom doping. A topological discussion suggests that simple 3 -polytopes with maximum numbers of inner diagonals close to the dopant may be good candidates of fullerene-like Si cages. First-principles calculation shows that, in the case of W doping, W@Si 12 and $\mathrm{W} @ \mathrm{Si}_{14}$ are energetically most favorable and also have the cages predicted by the above topological picture.

This work is partly supported by NEDO. 


\section{REFERENCES}

[1] A. A. Quong, M. R. Pederson and J. Q. Broughton, Phys. Rev. B 50, 4787 (1994); K. Jackson, G. Jungnickel and T. Fraunheim, Chem. Phys. Lett. 292, 235 (1998); V. Tozzini, F. Buda and A. Fasolino, Phys. Rev. Lett. 85, 4554 (2000).

[2] E. C. Honea et al., Nature (London) 366, 42 (1993).

[3] K. M. Ho et al., Nature (London) 392, 582 (1998).

[4] B.-x. Li, P.-l. Cao and D.-l. Que, Phys. Rev. B 61, 1685 (2000); see also references therein for previous theoretical studies of $\mathrm{Si}_{60}$.

[5] E. Kaxiras, Phys. Rev. Lett. 64, 551 (1990); U. Roethlisberger, W. Andreoni and M. Parrinello, ibid. 72, 665 (1994); J. Pan and M. Ramakrishna, Phys. Rev. B 50, 15431 (1994).

[6] J. L. Elkind et al., J. Chem. Phys. 87, 2397 (1987).

[7] M. F. Jarrold, Science 252, 1085 (1991); M. F. Jarrold and V. A. Constant, Phys. Rev. Lett. 67, 2994 (1991); E. Kaxiras and K. Jackson, ibid. 71, 727 (1993); L. Mitas, J. C. Grossman, I. Stich and J. Tobik, ibid. 84, 1479 (2000).

[8] H. Hiura, T. Miyazaki and T. Kanayama, Phys. Rev. Lett. 86, 1733 (2001).

[9] H. Kawaji et al., Phys. Rev. Lett. 74, 1427 (1995).

[10] S. Wei R. N. Barnett and U. Landman, Phys. Rev. B 55, 7935 (1997); F. Hagelberg, I. Yanov and J. Leszczynski, J. Mol. Struct. (THEOCHEM) 487, 183 (1999); C. Xiao and F. Hagelberg, ibid. 529, 241 (2000); J.-G. Han and Y.-Y. Shi, Chem. Phys. 266, 33 (2001); J.-G. Han and F. Hagelberg, ibid. 263, 255 (2001); V. Kumar and Y. Kawazoe, Phys. Rev. Lett. 87, 045503 (2001).

[11] S. M. Beck, J. Chem. Phys. 87, 4233 (1987); ibid. 90, 6306 (1989).

[12] D. Bremner and V. Klee, J. Combin. Theory - Ser. A 87, 175 (1999).

[13] A "simple 3-polytope" is a polytope in 3 dimension where each vertex is incident to precisely three edges. A 3-polytope is called "simplicial" if each of its facets is a triangle. For general properties of polytopes, see, for example, G. M. Ziegler, Lectures on Polytopes (Springer, 1998).

[14] It is cautioned that the Si cage structures are not necessarily convex simple 3-polytopes, especially in high-energy structures. Some of them are not even convex. In spite of this, one can model well low-energy structures with convex simple 3-polytopes.

[15] Suppose that $P$ has $E$ edges and $F$ facets, then $E=\left(4 N_{4}+5 N_{5}+6 N_{6}\right) / 2$ and $F=N_{4}+$ $N_{5}+N_{6}$. Since $P$ is simple, $E=3 n / 2$, meaning that $n$ must be even. Combining these with the Euler's formula $n+F=E+2$, we get $2 N_{4}+N_{5}=12$ and $N_{5}+2 N_{6}=n-8$. Note that $P$ 's with $\vec{N}=(6,0,1)$ and $(5,2,1)$ do not exist. The two conditions obtained here are necessary but not sufficient for the existence of $P$.

[16] Grünbaum and Motzkin have already clarified that, for $F \geq 14$, any simple 3-polytope with a maximum $\delta_{3}$ is composed of only twelve pentagons and $F-12$ hexagons. See, B. Grünbaum and T. S. Motzkin, Canad. J. Math. 15, 744 (1963).

[17] I. Rata et al., Phys. Rev. Lett. 85, 546 (2000).

[18] M. J. Frisch et al., Gaussian 98, Revision A.7 (Gaussian, Pittsburgh, PA, 1998).

[19] Each cluster is located in a simple cubic supercell with the edge length of 30 a.u. in PW calculation. The self-consistent field iterations are continued until the total energy converges to $\sim 3 \times 10^{-6} \mathrm{eV} /$ atom. Only a $\Gamma$ point is sampled for Brillouin-zone integration. 
The atomic positions of all atoms in supercell are relaxed until the residual forces are less than $\sim 0.06 \mathrm{eV} / \AA$.

[20] STATE (Simulation Tool for Atom TEchnology), Version 3.50, Joint Research Center for Atom Technology (JRCAT), May 2000.

[21] See, for example, M. C. Payne et al., Rev. Mod. Phys. 64, 1045 (1992) and references therein.

[22] A. D. Becke, Phys. Rev. A 38, 3098 (1988).

[23] J. P. Perdew and Y. Wang, Phys. Rev. B 45, 13244 (1992).

[24] J. P. Perdew, K. Burke and M. Ernzerhof, Phys. Rev. Lett. 77, 3865 (1996).

[25] Coulomb interactions between electrons and ions are approximated with effective-core potentials [27] (for LCAO) and pseudopotentials (PP's; for PW). In PW calculation, PP's of W and Si are as follows; For W, $5 p, 5 d$ and $6 s$ states are considered, where $5 p$ and $5 d$ orbitals are pseudized with ultra-soft PP's [28] and $6 s$ with a norm-conserving PP (NCPP) [29]. Only NCPP's are constructed for $3 s$ and $3 p$ states of Si.

[26] L. Gmelin et al., Gmelin Handbook of Inorganic and Organometallic Chemistry, 8-th Edition, W Suppl. A 5b, 155 (Springer-Verlag, Berlin, 1993).

[27] P. J. Hay and W. R. Watt, J. Chem. Phys. 82, 270 (1985); W. R. Watt and P. J. Hay, ibid. 82, 284 (1985).

[28] K. Laasonen et al., Phys. Rev. B 47, 10142 (1993).

[29] N. Troullier and J. L. Martins, Phys. Rev. B 43, 1993 (1991). 


\section{TABLES}

TABLE I. Topological properties and energetics of $\mathrm{W} @ \mathrm{Si}_{n}$ clusters. For definitions of $n, \vec{N}$, $\delta_{3}, \delta_{3}^{e f f}$ and $d_{\text {cut }}$, see text. $d_{1}$ and $d_{2}$ are the Si-W and Si-Si distances in $\AA$, averaged over the respective pairs whose separations are less than $2.7 \AA . \Delta E$ is the total energy ( $\mathrm{eV}$ ) relative to that of the lowest-energy cluster, $\vec{N}=(6,0,2)$ for $n=12$ and $\vec{N}=(3,6,0)$ for $n=14$.

\begin{tabular}{|c|c|c|c|c|c|c|c|c|c|c|c|}
\hline \multirow[t]{2}{*}{$n$} & \multirow[t]{2}{*}{$\vec{N}$} & \multirow[t]{2}{*}{$\delta_{3}$} & \multicolumn{5}{|c|}{$\delta_{3}^{e f f}\left(d_{\text {cut }}\right)$} & \multirow[t]{2}{*}{$d_{1}$} & \multirow[t]{2}{*}{$d_{2}$} & \multirow[t]{2}{*}{ structure } & \multirow[t]{2}{*}{$\Delta E$} \\
\hline & & & $d_{\text {cut }}(\AA)=$ & 1.0 & 0.6 & 0.3 & 0.25 & & & & \\
\hline$\overline{12}$ & $(4,4,0)$ & 20 & & 8 & 4 & 0 & 0 & 2.59 & 2.42 & Fig.1 (c) & 2.16 \\
\hline 12 & $(6,0,2)$ & 18 & & 6 & 6 & 6 & 6 & 2.67 & 2.39 & Fig.1 (d) & 0.00 \\
\hline$\overline{14}$ & $\overline{(3,6,0)}$ & 34 & & 4 & 4 & 4 & 4 & 2.69 & 2.34 & Fig.1 (e) & 0.00 \\
\hline 14 & $(4,4,1)$ & 33 & & 11 & 5 & 1 & 0 & 2.66 & 2.35 & Fig.1 (f) & 0.95 \\
\hline 14 & $(5,2,2)$ & 32 & & 13 & 4 & 2 & 2 & 2.64 & 2.39 & Fig.1 (g) & 0.63 \\
\hline 14 & $(6,0,3)$ & 31 & & 9 & 7 & 4 & 0 & 2.63 & 2.39 & Fig.1 (h) & 0.89 \\
\hline
\end{tabular}




\section{Figure captions}

Figure 1 (color) Calculated structures of $\mathrm{W}_{\mathrm{S}} \mathrm{Si}_{n}$ in "fullerene-like" cages. Double line segments are drawn when the inter-atom distance is less than $2.7 \AA$. The double line segments corresponding to the edges of simple 3-polytopes are colored with blue. In panels (a), $(\mathrm{g})$ and $(\mathrm{h})$, red single lines should be supplemented and also green double lines should be removed to retrieve connectivity in the corresponding simple 3-polytopes. See also a note [14].

Figure 2 (color) Calculated structures of $\mathrm{W} @ \mathrm{Si}_{16}$. Si atoms colored with dark blue are regarded "attached" to either the (6,0,2)-cage $\mathrm{W} @ \mathrm{Si}_{12}$ (panel (e)) or (3,6,0)-cage $\mathrm{W} @ \mathrm{Si}_{14}$ clusters (panels (a), (b), (c) and (d)). Other conventions are the same as in Fig.1. 